\title{
Suivi et diagnostic médicaux: deux jugements récents du Tribunal fédéral
}

\section{Valérie Junod}

Professeure de droit aux Universités de Genève et de Lausanne

\footnotetext{
* Les références se trouveront sous www.bullmed.ch

$\rightarrow$ Numéro actuel ou

$\rightarrow$ Archives $\rightarrow 2012 \rightarrow 40$.
}

Correspondance:

Prof. Dr iur. Valérie Junod UNIL, Ecole des HEC Internef 615

CH-1015 Lausanne Tél. 0216923428

Fax 0216923305

valerie.junod[at]unil.ch
Le Tribunal fédéral a jugé en mai deux affaires concernant la responsabilité du médecin pour un diagnostic non posé, respectivement mal posé. Le Tribunal y retrace, puis affine, les principes importants du droit médical. Dans les deux cas, les médecins sont exonérés de toute responsabilité, tandis que les familles des deux patients décédés sont déboutées. Ces deux arrêts rappellent que gagner un procès contre un médecin est loin d'être facile - malgré les craintes de condamnations si souvent exprimées par les professionnels de la santé.

La présente note relate les principaux faits à la base des deux cas, puis résume la motivation articulée par le Tribunal fédéral [1]*. La conclusion dégage les enseignements importants pour les médecins.

\section{Les faits à la base des deux affaires}

Dans le premier cas tranché le 2 mai 2012 (4A_737/2011), le patient, un homme né en 1925, avait consulté à de nombreuses reprises un urologue, le docteur A, pour une prostate suspectée de malignité. Entre juin 1993 et octobre 1995, le médecin avait procédé ou fait procéder à de multiples tests, contrôles et traitements (biopsie, prises de sang pour calcul du taux PSA, médicaments, résection de la prostate). Aucun de ces tests n'avait révélé de cancer, mais la forme et la sensibilité de la prostate continuaient à préoccuper le patient et son médecin. Lors du dernier rendez-vous d'octobre 1995, le médecin disait avoir avisé son patient qu'il lui fallait prendre rendez-vous avec un urologue spécialisé à l'hôpital en vue d'un suivi plus sophistiqué; le médecin affirmait avoir contacté par téléphone ce spécialiste pour l'informer du dossier. Pour sa part, le patient niait avoir reçu cette recommandation; au contraire, il considérait que le médecin l'avait abandonné à son sort. De fait, il n'y avait eu aucune visite médicale entre mi-octobre 1995 et fin février 1999, où le patient s'était rendu chez un autre médecin qui avait diagnostiqué un cancer prostatique avec métastases multiples. Le patient avait été traité à l'hôpital par d'autres médecins, mais était décédé de son cancer quelques années plus tard en avril 2004.

Dans la seconde affaire tranchée le 23 mai 2012 (4A_760/2011), un homme né en 1951, fumeur, alcoolique et souffrant d'hypertension, avait ressenti de vives douleurs à la poitrine et au bras droit dans la nuit du jeudi 23 au vendredi 24 mai 2002. Il avait immédiatement consulté son médecin traitant le Dr B, ce dernier n'ayant cependant pu le recevoir qu'à $17 \mathrm{~h}$ vendredi 24 mai. Comme le patient disait ne pas éprouver de sensation d'étau dans la cage thoracique, ne pas ressentir de manque d'air et ne pas avoir de douleurs thoraciques en marchant, le Dr B avait exclu un infarctus. Il n'avait toutefois pas effectué d'électrocardiogramme. Par ailleurs, il avait noté des râles crépitants du poumon droit, tandis que la radiographie du poumon révélait une légère opacité; le médecin avait en conséquence diagnostiqué une bronchite virale et avait prescrit des antibiotiques et des anti-inflammatoires. Le samedi, le Dr B avait appelé son patient qui lui avait signalé une légère amélioration, les douleurs ayant diminué. Un nouveau rendez-vous avait été fixé pour lundi. Cependant, dans la nuit de samedi à dimanche, l'état du patient s'était nettement détérioré avec des douleurs thoraciques irradiantes. Malgré les pressions de sa famille pour qu'il se rende aux urgences dans la nuit, le patient avait refusé. Lors du rendez-vous de lundi, le Dr B avait diagnostiqué un infarctus antérieur étendu. Transféré de suite à l'hôpital, le patient y était décédé ce même lundi d'une tamponnade cardiaque [2] pendant une coronarographie.

\section{Le raisonnement du Tribunal fédéral}

Le Tribunal fédéral devait déterminer si les jugements cantonaux qui déboutaient les familles des deux patients décédés étaient conformes au droit fédéral. Ces familles ayant perdu devant les juridictions cantonales, elles se trouvaient d'emblée en position défavorable devant le Tribunal fédéral, lequel ne revoit en principe pas les faits retenus par l'instance cantonale, mais seulement son application du droit.

\section{L'arrêt du 2 mai 2012}

Le Tribunal commence par rappeler les conditions ultra-classiques que le patient, qui veut mettre en cause la responsabilité contractuelle de son médecin, doit prouver, à savoir:

- l'existence d'un contrat entre lui-même et son médecin (généralement un mandat),

- la violation d'une obligation issue de ce contrat par le médecin,

- la survenance d'un dommage ou d'un tort moral,

- un lien de causalité naturelle et adéquate entre cette violation et ce dommage ou tort moral.

Si le patient parvient à établir ces quatre conditions, le médecin peut encore se disculper en prouvant qu'il n'a commis aucune faute - ce qui, en pratique, est quasiment impossible. 


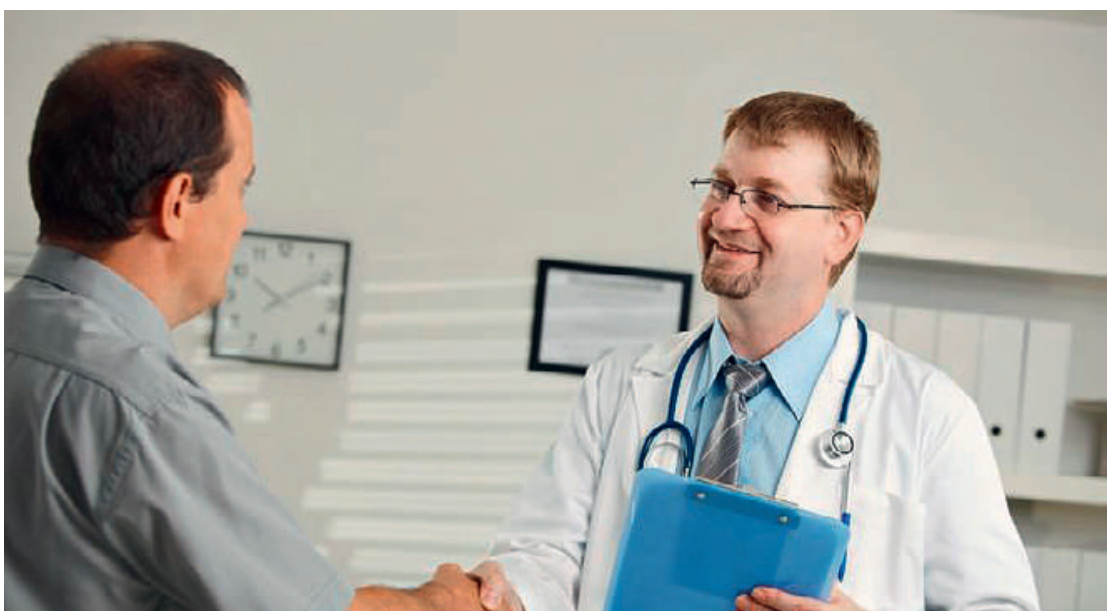

Le médecin affirmait avoir recommandé de prendre rendez-vous avec un urologue spécialisé à l'hôpital. Le patient le niait.

Le patient supporte un lourd fardeau de la preuve. En particulier, c'est lui qui doit identifier et prouver la violation du contrat, laquelle consiste soit en un manquement au devoir de diligence du médecin apprécié au regard des règles de l'art médical, soit en une intervention effectuée sans le consentement libre et éclairé du patient. Souvent, les patients préfèrent invoquer l'absence de consentement de leur part, car le fardeau de la preuve s'en trouve allégé. En effet, c'est alors au médecin d'apporter la preuve qu'il a bel et bien obtenu le consentement du patient avant toute intervention sur son corps.

Ici, la famille du patient décédé estimait qu'il appartenait au médecin de prouver avoir dûment informé son patient, en particulier sur la nécessité de prendre contact avec le spécialiste de l'hôpital. Autrement dit, de son point de vue, le fardeau de la preuve devait reposer sur le médecin, et non sur elle.

\section{Ce n'est que lorsque le médecin porte atteinte au corps du patient que la preuve du consentement préalable incombe au médecin.}

Le Tribunal fédéral lui donne tort. Ce n'est que lorsque le médecin porte atteinte au corps du patient, notamment par une intervention, que la preuve du consentement préalable incombe au médecin. En effet, dans cette hypothèse, le médecin touche à l'intégrité corporelle du patient et cette atteinte est automatiquement illicite, sauf à prouver le consentement du patient. Il n'en va pas de même si le médecin n'intervient aucunement sur le corps de son patient. Dans le cas présent, lors du rendez-vous d'octobre 1995, aucune intervention n'avait eu lieu, uniquement une discussion (au contenu litigieux s'agissant du suivi médical ultérieur). Dès lors, le médecin n'avait pas à prouver avoir obtenu le consente- ment du patient sur les modalités de suivi; au contraire, il incombait au patient de prouver une violation du contrat, notamment un manquement du médecin à son devoir d'information.

Par conséquent, les juges devaient apprécier si la famille du défunt avait apporté la preuve que le Dr A avait abandonné son patient à son sort, se désintéressant de son suivi médical. Le médecin et le centre de soins pour lequel il intervenait avaient produit un dossier médical et des notes personnelles desquels il ressortait que le médecin avait bel et bien recommandé au patient de consulter le spécialiste de l'hôpital. De son côté, la famille du défunt n'avait pas pu produire la moindre preuve écrite. Quant au spécialiste recommandé à l'hôpital, quatorze ans plus tard, il n'avait gardé aucun souvenir du coup de fil du Dr A. Le Tribunal fédéral devait donc juger si la version des faits exposée par la famille était hautement vraisemblable ou si c'était à raison que les tribunaux cantonaux avaient préféré la version du médecin. A ce stade, la motivation du Tribunal verse dans l'analyse psychologique:

«Dès lors qu'il n'y avait aucune certitude et que les analyses du PSA étaient plus alarmantes, on ne peut pas imaginer que l'urologue [le Dr A], lors du rendez-vous du 17 octobre 1995, n'ait proposé à son patient ni examen plus approfondi, ni traitement, ni même un rendez-vous de contrôle quelque temps plus tard. [...] Si l'on suppose, avec le recourant, que l'urologue [Dr A] aurait décidé de se désintéresser de son patient et de l'abandonner à son sort, on ne comprend pas que le patient, décrit dans le recours comme un homme intelligent, soucieux de sa santé et ainsi de détecter un cancer de manière précoce, soit resté passif pendant plus de trois ans.»

Pour le Tribunal, l'explication proposée par le Dr A est plus logique: le patient avait déjà subi deux interventions médicales (une biopsie et une résection) avec des résultats négatifs, ne souffrait actuellement plus et dès lors voulait éviter de «subir, en milieu hospitalier, des actes médicaux invasifs, peut-être douloureux ou mutilants». Son choix d'attendre un peu avant de consulter à nouveau tout en observant «lui-même l'évolution de son état» n'était «ni insensé, ni déshonorant», mais «au contraire humainement compréhensible». Le Tribunal fédéral profite pour rappeler que: «dans le domaine médical, [il] appartient au patient de prendre rendez-vous, puisqu'il reste libre de décider de ne pas se soigner, de choisir le médecin qu'il veut consulter et de déterminer s'il veut ou non solliciter des prestations médicales qui entraîneront des dépenses pour lui.»

Sans commenter ces aspects, le Tribunal admet qu'après avoir recommandé au patient de prendre rendez-vous avec le spécialiste de l'hôpital, le Dr A n'était plus tenu de suivre son patient en parallèle. De plus, le médecin n'a pas à exercer de pressions sur son patient pour que ce dernier prenne effectivement le rendez-vous recommandé. Il n'était pas non 
plus impératif qu'il contacte lui-même le spécialiste, du moment qu'il avait dit à son patient de le faire. Par conséquent, c'est à raison que la famille du défunt a été déboutée par les instances cantonales, puisqu'elle n'est pas parvenue à prouver que le Dr A aurait manqué à ses obligations.

\section{L'arrêt du 23 mai 2012}

Dans cette seconde affaire, suite au décès du patient, le Dr B avait spontanément admis s'être trompé dans son diagnostic le vendredi 24 mai 2002 et ne s'en être rendu compte que trop tard le lundi 27 mai 2002 [3]. Compte tenu des facteurs de risque du patient, le médecin aurait dû procéder à un électrocardiogramme. Ce point n'étant plus controversé, les tribunaux avaient pu retenir un manquement à la diligence appréciée à l'aune des règles de l'art médical. Restait à déterminer si cette faute de diagnostic [4] était la cause naturelle et adéquate du décès du patient (plus exactement du dommage résidant dans la perte de soutien financier subie par sa famille). Cela revenait à se demander si le décès aurait pu être évité si le Dr B avait correctement diagnostiqué l'infarctus vendredi soir. La preuve de cette condition incombe aux demandeurs, à savoir la famille du défunt. Comme il est très difficile d'établir avec certitude comment l'état du patient aurait évolué si le diagnostic correct avait d'emblée été posé (causalité dite hypothétique), le degré de preuve requis des demandeurs est abaissé au niveau de la vraisemblance prépondérante. Une expertise médicale est pratiquement indispensable pour établir ce qui se serait très vraisemblablement produit si le patient avait été traité immédiatement vendredi soir pour son infarctus.

\section{«Les professionnels de la santé ont tout intérêt à noter soigneusement les discussions tenues avec leurs patients et les mesures décidées.»}

Or ici l'expert mandaté par le tribunal cantonal avait été très hésitant. Il estimait qu'un individu subissant un infarctus a les meilleures chances d'éviter la perforation ventriculaire (tamponnade avec rupture myocardique) s'il est pris en charge dans les 3 heures suivant l'infarctus; entre 6 et 12 heures, les résultats sont encore acceptables; après 12 heures, il est très rare qu'ils le soient encore, sans que cela puisse être absolument exclu.

Pour les juges, cette formulation de l'expertise ne permet pas d'affirmer, avec un degré de vraisemblance prépondérante, que le décès du patient aurait pu être évité si le Dr B avait diagnostiqué l'infarctus vendredi en soirée, car plus de 12 heures auraient séparé les premiers signes d'infarctus ressentis dans la nuit de jeudi à vendredi et la mise en route du traite- ment nécessaire, lequel aurait pu débuter au mieux après transfert à l'hôpital, soit dans la soirée du vendredi. Le Tribunal fédéral peut ainsi conclure que la famille du défunt a été déboutée à raison, n'étant pas parvenue à prouver la délicate condition de la causalité hypothétique.

\section{Conclusion}

Ces deux arrêts contiennent de nombreux enseignements pour les professionnels de la santé et les patients. On peut les résumer brièvement ci-après:

- Le dossier médical et les notes personnelles du médecin constituent un élément de preuve très précieux. Les professionnels de la santé ont tout intérêt à noter soigneusement les discussions tenues avec leurs patients et les mesures décidées [5].

- L'expertise judiciaire confiée à un médecin est très souvent décisive lorsqu'il s'agit de trancher entre deux visions médicales divergentes. L'expert doit être très soucieux du vocabulaire utilisé, l'issue du procès pouvant dépendre d'un simple qualificatif (par ex. hautement vraisemblable par opposition à possible) [6] .

- Lorsque les versions divergentes des parties ne portent pas sur des faits médicaux, mais sur leurs déclarations et leurs agissements, les juges peuvent raisonner en termes de logique comportementale. Ainsi, ils peuvent retenir qu'un médecin normal n'aurait pas «oublié» un patient dont la prostate suscitait de fortes inquiétudes et qu'un patient normalement intelligent [7] n'aurait pas «oublié» de faire suivre sa prostate, à moins d'une volonté délibérée «de couper les ponts avec le monde médical pendant un certain temps» [8]. Cet exercice de psychologie a des fondements fragiles (ici les juges insistent beaucoup sur l'intelligence du patient), rendant l'issue du procès d'autant plus aléatoire.

- Le patient peut, mais aussi doit, exercer de manière intelligente et prudente son droit à l'autodétermination. Les juges estiment qu'un patient adulte doté de moyens intellectuels normaux doit se préoccuper de son état de santé, et ne peut s'abstenir de consulter pendant de nombreuses années (arrêt du 2 mai 2012) ou en cas d'urgence manifeste (arrêt du 23 mai 2012). C'est à lui que revient - en bonne partie - l'initiative de gérer sa santé.

Ces deux affaires rappellent qu'il n'est guère aisé de mettre en cause la responsabilité du médecin. Les preuves que les demandeurs doivent apporter sont difficiles à réunir. Contrairement aux professionnels de la santé, les patients ne prennent pas de notes et ne tiennent pas de dossier. Ils dépendent des conclusions de l'expert médical nommé par le juge. Cependant, dans la gestion de leur santé, il est attendu d'eux une attitude proactive. 